\title{
Enhanced Energy Deposition Efficiency of Glow Discharge Electron Beams for Metal Surface Treatment
}

\author{
Nélida Mingolo, Yanina Cesa, Oscar E. Martínez, Javier I. Etcheverry, and Jorge J. Rocca, Senior Member, IEEE
}

\begin{abstract}
The energy deposition efficiency and focal spot dynamics of electron beams produced by pulsed cold-cathode highvoltage glow discharges for metal surface treatment are investigated for two different cathode geometries. A concave cathode geometry in which the focusing is dominated by the convergence of the electric field lines in the cathode fall region is compared with a flat cathode in which the focusing is exclusively caused by the self-generated magnetic field. Results of the treatment of AISI 4140 carbon steel samples show that the concave cathode geometry significantly increases the efficiency, reduces the threshold power necessary for melting, and is less sensitive to variations in the discharge parameters and sample position. The results of numerical modeling indicate that the observed increase in efficiency is caused by the longer persistence of the focal spot on the sample. The model can be used to predict the discharge parameters required for a desired treatment.
\end{abstract}

Index Terms-Glow discharge, electron gun, surface treatment.

\section{INTRODUCTION}

$\mathbf{T}$ HE surface treatment of metallic surfaces by means of energetic beams is a well-established technique [1], [2]. In particular, laser surface melting has proved a valuable tool for changing the structure of a layer of the material by solidification after rapid cooling toward the substrate [3]-[10]. On the other hand, continuos wave electron beams have been used for annealing purposes [11]-[14]. The use of energetic electron beam pulses generated by cold-cathode glow discharges [15] presents an alternative processing method, which allowed for single-shot surface amorphization of a well-known glass forming alloy. In previous work, Mingolo et al. used a modified version of a flat cathode electron gun originally designed for plasma excitation [16]. These electron beams had energies in the tens of kilovolts, and currents of tens of amperes that create a magnetic field forcing the beam to collapse. In those experiments with magnetic self-focusing, the treated area was selected by the cathode-sample distance. Besides their simplicity and low cost,

Manuscript received June 10, 1999; revised October 12, 1999. This work was supported in part by the Universidad de Buenos Aires, CONICET, and the Agencia Nacional de Promoción Cientifica y Tecnológica.

N. Mingolo and Y. Cesa are with the Laboratorio de Haces Dirigidos, Departamento de Física, Facultad de Ingeniería, Universidad de Buenos Aires, 1063 Buenos Aires, Argentina (e-mail: nmingol@tron.fi.uba.ar).

O. E. Martínez and J. I. Etcheverry are with the Facultad de Ciencias Exactas y Naturales, Universidad de Buenos Aires, Ciudad Universitaria, 1428 Buenos Aires, Argentina.

J. J. Rocca is with the Department of Electrical Engineering, Colorado State

University, Fort Collins, CO 80523 USA.

Publisher Item Identifier S 0093-3813(00)03663-8. the other potential advantages of this type of guns for surface treatment include the easily obtainable large energy per shot (tens of Joules), the lower reflectivity and the larger penetration of the electrons in metals as compared with light, and a depth profile that does not peak at the surface [17], [18]. Despite these advantages, several problems develop with the flat cathode design: the position of the focus, and hence, the size of the spot at the sample, are extremely sensitive to the current. The shot-to-shot reproducibility was improved significantly by adding a continuos low current discharge to the capacitive discharge producing the electron beam pulse [19]. Despite these improvements, the beam still rapidly changes its focal point as the discharge evolves, because of the decrease of the current, and, hence, in the focusing magnetic field. This dynamic evolution of the spot size gives rise to short, effective pulsewidths, as most of the time the beam is defocused, which causes a major reduction in the energy deposition efficiency $\varepsilon$, defined as the electron beam energy deposited in the central spot of the sample divided by the energy stored by the capacitor, losing one of the major potential advantages for surface treatment. Also, the beam size changes rapidly with the distance to the focus location, which gives rise to the need of a precise location of the sample for a given beam size. It is shown herein that these two limitations can be ovecome with the use of a concave cathode geometry.

In this work, a curved cathode geometry is investigated and the results are compared with those corresponding to a flat cathode. The concave cathode geometry introduces an additional focusing mechanism that is the result of converging electric field lines in a dark space region, thus providing a geometric focusing mechanism competing with the magnetic self-focusing. This mechanism is in contrast with the case of a flat cathode, in which the focusing is exclusively caused by the self-generated magnetic field. This cathode geometry was previously used for low-current continuos wave discharges by Rocca et al. [20], [21] in situations in which the magnetic force could not provide enough focusing action. In the curved cathode pulsed discharge, the magnetic focusing is still present, and the spot size at the sample results from the combined action of both mechanisms. It is shown that with the adequate design, the geometric mechanism can stabilize the beam size in such a manner that a large increase in the efficiency of the gun is obtained.

In the following section, the characteristics of the curve cathode geometry are described, followed by a discussion of the numerical simulations allowing the prediction of the increase 
in the stability of the focus location, and the determination of the operating conditions under which the geometric focusing mechanism prevails over the magnetic self-focusing. Section IV presents the experimental results obtained using AISI 4140 carbon steel samples, including the melting thresholds corresponding to each configuration. The origin of the difference in efficiency is discussed in Section V.

\section{Characteristics of THE Curved CATHOde GeOMEtRy}

In a low-pressure high-voltage glow discharge, the geometry of the cathode can be used to shape the trajectory of the beam electrons within the cathode fall region, where the overwhelming majority of the discharge voltage drop occurs. This result is possible because within a selected range of discharge pressures, the equipotential lines follow the shape of the cathode surface in practically the entire cathode fall region. Evidence of this effect can be readily visually observed in direct-current high-voltage glow discharges in which the boundary between the cathode dark space and the negative glow can be seen to approximately follow the shape of the cathode surface. In the particular case of a concave cathode geometry, this results in radial components of the electric field accelerating the electrons toward a focal point. It should be noticed that the generation of focused high-energy electron beams by this effect can only be obtained within a selected pressure range. At these pressures, it is possible to simultaneously obtain a high-cathode fall voltage (i.e., kilovolts), and a cathode fall region thickness that is small compared with the cathode diameter. The thickness of the cathode fall region in low-pressure cold-cathode glow discharges follows the well-known scaling law [22], [23] $p \cdot d \approx C$, where $d$ is the thickness, $p$ is the pressure, and $C$ is a constant that depends mainly on the type of gas. Therefore, if the pressure is excessively low, the thickness of the cathode fall region grows up to a point at which the shape of equipotential lines within the entire dark space is no longer determined by the geometry of the cathode surface. On the contrary, if the pressure is excessively high, the equipotential lines closely follow the shape of the cathode surface, but the voltage drop across the cathode fall region is insufficient to accelerate the electrons to a high energy. Nevertheless, if the pressure is adequately selected (e.g., 1 torr for a He discharge with a cathode several centimeters in diameter), this geometric focusing mechanism provides a simple scheme to generate tightly focused electron beams.

A schematic of the curved cathode gun is given in Fig. 1(a). As the typical focal distances of the flat cathode design varied between $35 \mathrm{~cm}$ for high current pulses to $60 \mathrm{~cm}$ for the less energetic treatments, a cathode radius of curvature of $20 \mathrm{~cm}$ was chosen. In fact, the cathode radius of curvature should be shorter than the magnetic focusing distance for the geometrical mechanism to prevail. On the other hand, the radius of curvature should be larger than the cathode radius to stay within the small angle approximation required if the gun is to work in the geometrical approach used here. The cathode radius was $r_{o}=31 \mathrm{~mm}$, as compared with $r_{o}=35 \mathrm{~mm}$ used in the plane cathode experiments. In the present experiments, besides changing the cathode radius of curvature, a current-limiting inductance of $180 \mu \mathrm{H}$ was
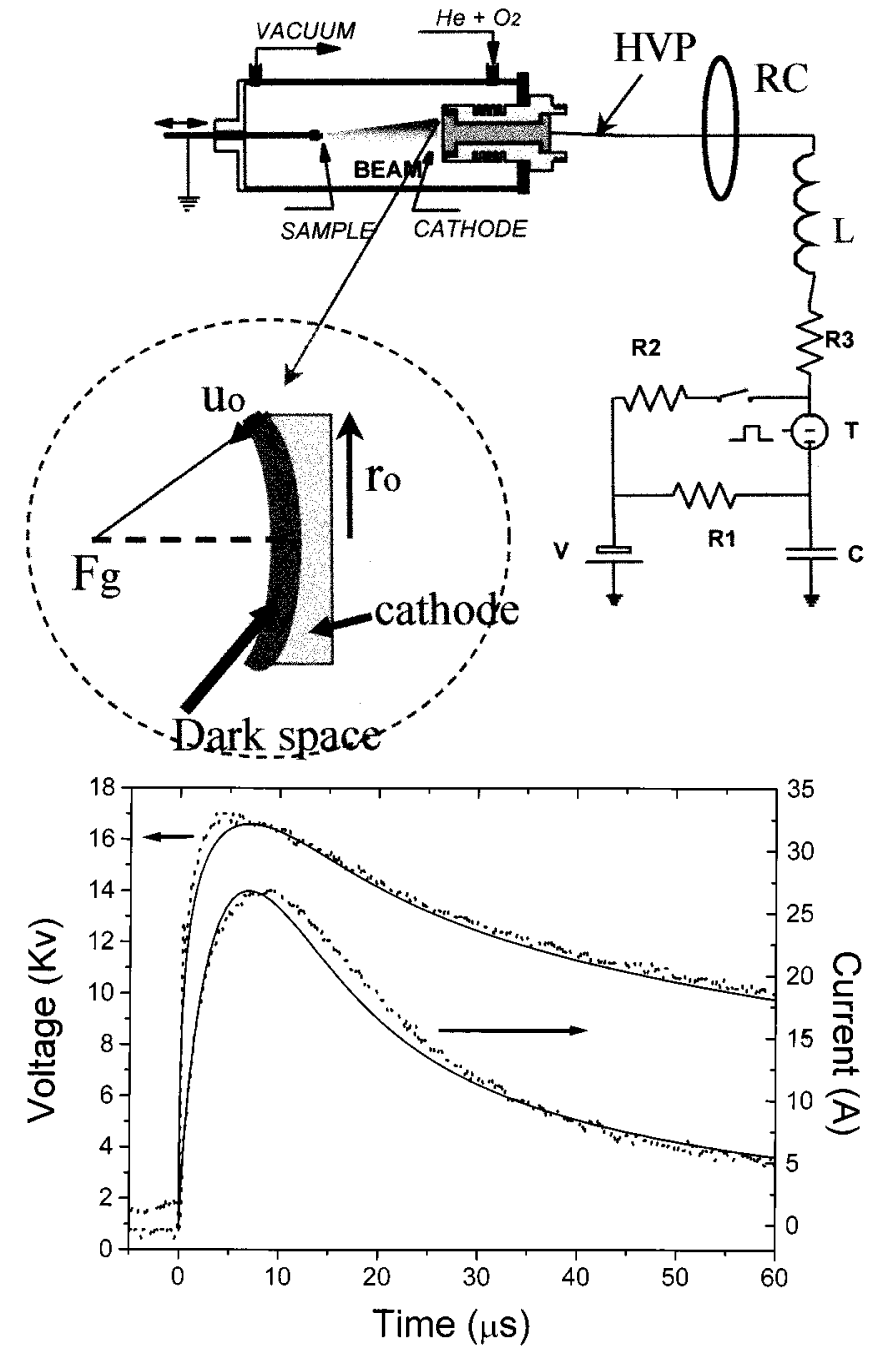

Fig. 1. (a) Schematic diagram of the electron gun and discharge circuit. The "keep alive" current is kept through $R_{2}$, and the main discharge of capacitor $C$ is set by the trigger $T$, through a limiting resistor $R_{3}$ and the inductance $L=180 \mu \mathrm{F}$ added in this work. A Rogowski coil (RC) and a high voltage probe (HVP) monitor the discharge. The inset shows the details of the curved cathode, $F_{g}$ is the geometrical focal distance (equal to the cathode radius of curvature), $r_{o}$ is the cathode radius, and $u_{o}$ is the initial electron velocity. (b) Voltage and current pulses. Full lines: numerical simulations solving the circuit using the phenomenological law for the discharge given in (1). Dots: experimental results. Similar agreements are found for all shots.

added that slightly rounds the current peak and limits eventual arcs that may develop.

\section{Numerical Simulation of THE TRANSIENT Defocusing}

Numerical simulations of the discharge and focusing action of the beam were carried out using the same differential equations given by Etcheverry et al. [18]. The analytical solutions found previously [18] for the temporal evolution are no longer valid because of the added inductance. The equations used are

$$
\begin{gathered}
I=b V^{3} \\
\frac{d^{2} r}{d t^{2}}=-\frac{\mu_{0} I e}{2 \pi m r} \frac{d z}{d t} \\
\frac{d^{2} z}{d t^{2}}=\frac{\mu_{0} I e}{2 \pi m r} \frac{d r}{d t} .
\end{gathered}
$$



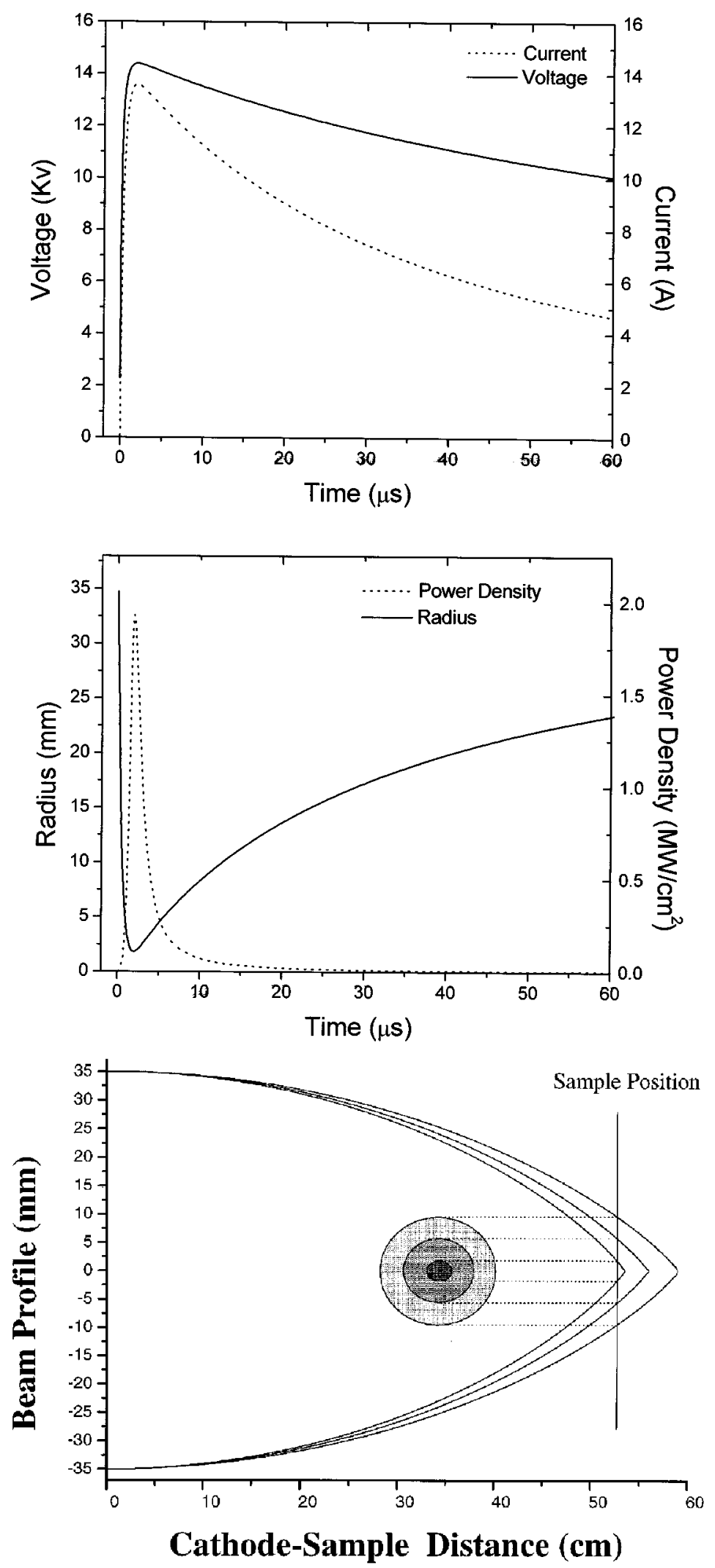

Fig. 2. Numerical simulation of the beam evolution for a plane cathode case. The capacitor initial voltage is $15 \mathrm{kV}$. The sample is located $53 \mathrm{~cm}$ away from the cathode to produce a minimum spot size of $1.8 \mathrm{~mm}$. (a) Time evolution of the voltage, current, beam size, and power density at the sample. (b) Beam spatial profile at three different times: at the peak current, after a current drop of $10 \%$, and after a current drop of $20 \%$. The insert shows the corresponding spot sizes.

$V$ is the cathode voltage, $I$ is the beam current, and $b$ is a constant characterizing the gun depending on the cathode surface and the gas pressure. The constants $e$ and $m$ are the electron charge and mass, $r$ is the radial position of the electron from 
the beam axis, $z$ is the distance from the cathode, and $\mu_{0}$ is the vacuum magnetic permeability. The first equation originates from an empirical fit for different experimental situations [16], [18]. In Fig. 1(b), the fit of a typical experimental trace of the voltage and current pulses is shown using the empirical relation given in (1), showing a good agreement. The other two equations correspond to the motion of an electron emitted from the cathode border $r=r_{0}($ at $z=0)$, with initial speed $u_{0}=$ $(2 \mathrm{eV} / \mathrm{m})^{1 / 2}$, and direction perpendicular to the cathode surface, as indicated in the inset of Fig. 1(a).

The main assumption here is that the voltage drop occurs in the cathode sheath, about $1 \mathrm{~cm}$ wide. Hence, the geometric approximation used will remain valid as long as the radius of curvature remains long compared with the cathode radius (small angle). The effects of collisions of the beam electrons with the gas atoms beyond the cathode sheath is neglected, as proved adequate in prior works [16], [18].

To illustrate the effect of the cathode curvature, two numerical simulations are presented for plane and curved cathodes, with identical initial voltages, temporal evolution of the discharge, and initial beam size at the sample position. In Fig. 2(a), the temporal evolution of the relevant parameters is illustrated for the plane cathode case. The voltage drop across the glow discharge peaks close to the $15-\mathrm{kV}$ value of the initial capacitor voltage. The added inductance prevents the rapid voltage rise observed in our prior works [17]-[19]. It can be noticed that the beam current drops faster than the voltage, because of the power dependence shown in (1). The beam radius reaches a minimum of $1.8 \mathrm{~mm}$ at the current peak value of $13.6 \mathrm{~A}$, but rapidly broadens by a factor of more than five when the current drops only $20 \%$ $12 \mu \mathrm{s}$ after the trigger. The last plot shows the power density evolution.

Fig. 2(b) shows the beam spatial profile at three different times: at the current peak $(t=2 \mu \mathrm{s})$, after a $10 \%$ drop of the current $(t=6.5 \mu \mathrm{s})$, and after a $20 \%$ drop of the current $(t=12 \mu \mathrm{s})$. The position of the sample is indicated, as well as the three spots. The more rapid decay of the current as compared with the voltage, together with the high nonlinearity of the focusing action, is the origin of the fast broadening of the beam.

The case of a curved cathode with $20-\mathrm{cm}$ radius of curvature was computed for identical initial voltage and discharge parameters. The results (that also correspond to the experimental case shown later) are presented in Fig. 3. Obviously, the time evolution of the discharges are identical, but because of the geometrical focusing action, only a slight defocusing appears. The intensity at the center remains large for a longer period of time, increasing the efficiency. Observing the beam profile in Fig. 3(b) it can be noticed that the geometrical action yields a less nonlinear focusing (more conical), also reducing the sensitivity to target position. The spots sizes for the same times of Fig. 2(b) are shown, indicating the larger stability obtained. The key to the reduced sensitivity is that the geometrical focal distance $F_{g}$ is smaller than the magnetic focal distance $F_{m}$ obtained for the plane cathode under the same discharge conditions. The actual focal distance (about $16 \mathrm{~cm}$ ) for the curved cathode evolves toward the geometrical focal distance $(20 \mathrm{~cm})$ as the current drops to zero. Following similar steps as those of [14], but including the cathode curvature, it can be found that for $F_{g} / F_{m} \ll 1$, the actual focal distance $F$ results in

$$
F=F_{g}\left[1-2\left(F_{g} / F_{m}\right)^{2}\right]
$$

yielding the reduced sensitivity shown in the Fig. 3, even for the example given, where $F_{g} / F_{m}=0.4$.

\section{EXPERIMENTAL}

The samples used to test the improved performance of the curved cathode gun consisted of AISI4140 steel rods $40 \mathrm{~mm}$ in diameter and $5 \mathrm{~mm}$ thick. The samples were polished up to $1-\mu \mathrm{m}$ diamond powder and chemically etched with 5\% nital solution for metallographic observation in an optical microscope. Repolishing was performed before the electron gun treatment. Different discharge conditions were tested, and the samples were both treated by a single shot or repeatedly at $0.2 \mathrm{~Hz}$. The cathode sample distance was adjusted to obtain the desired spot size. The surfaces of the beam-irradiated samples were observed with an optical microscope and then etched with 5\% nital to reveal the grain structure.

In order to illustrate the differences in efficiencies between the curved and plane cathodes, different samples were treated with both configurations at different voltages and cathode-sample distances (i.e., different initial spot sizes). The gas pressure was set at 10 mtorr of $\mathrm{O}_{2}$ and $\mathrm{He}$ to complete 260 mtorr. For the plane cathode, the voltage was scanned between $12 \mathrm{kV}$ and $24 \mathrm{kV}$, the cathode-sample distance between $35 \mathrm{~cm}$ and $40 \mathrm{~cm}$, and the peak currents ranged between $15 \mathrm{~A}$ and $38 \mathrm{~A}$. The threshold for surface modification was found at $18.5 \mathrm{kV}$ and $27 \mathrm{~A}$ (peak powers of $500 \mathrm{~kW}$ ). The threshold was defined as the power at which a spot was observed by naked eye, but it was removed after the metallographic etching. When the curved cathode was introduced, surface changes were already observed with $12.5 \mathrm{kV}, 8 \mathrm{~A}$, yielding a threshold power of $100 \mathrm{~kW}$.

For a more precise quantitative comparison, two samples were selected that with the different cathode configurations, yielded similar results. For the curved cathode case, a sample was treated with a discharge of characteristics similar to that described in Fig. 3. The measured peak voltage and current were $15 \mathrm{kV}$ and $13.6 \mathrm{~A}$, yielding a peak power of $204 \mathrm{~kW}$. Precisely because of the difference in efficiency, the conditions described in Fig. 2 did not provide enough fluence (energy/area) to melt the sample (plane cathode). The voltage had to be increased, and the sample relocated until a spot was detected with a structure and size similar to that obtained with the curved cathode. This result was accomplished with $23.5-\mathrm{kV}$ cathode voltage and $35-\mathrm{cm}$ cathode-sample distance, yielding a peak power of $860 \mathrm{~kW}$. Fig. 4 shows the optical micrographs of the surfaces after chemical etching. Both samples presented a central spot about $4 \mathrm{~mm}$ in diameter with the structure shown and a wider area, with a slightly different structure corresponding to the region where the beam impacts as it defocuses. The two structures are similar and substantially different from the departing structure. The surface structure clearly shows signs of melting, and no particular grain structure appeared in the treated area after etching with 5\% nital (picral 

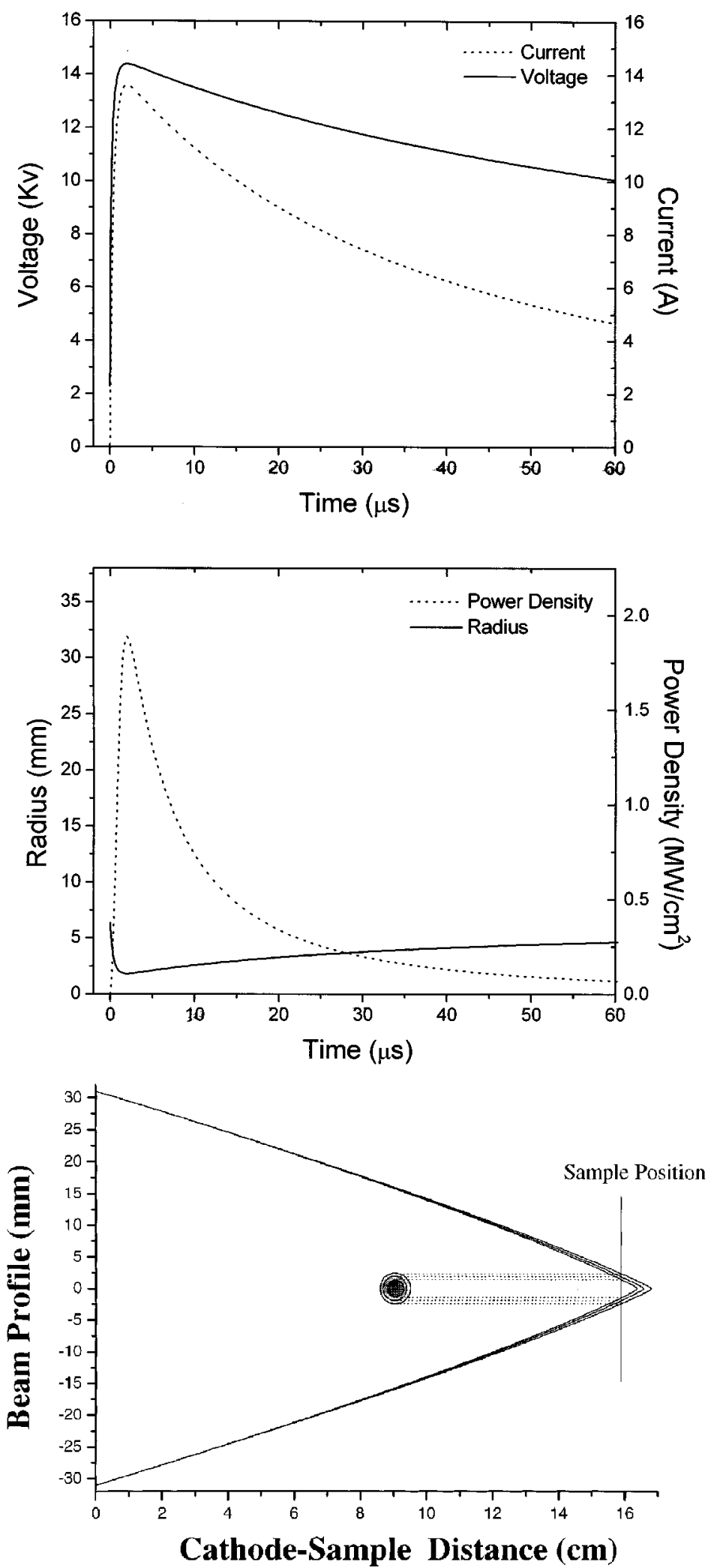

Fig. 3. Numerical simulation of the beam evolution for a curved cathode case, under identical initial conditions for the discharge as in Fig. 2. The sample is located $16 \mathrm{~cm}$ away from the cathode to produce the same minimum spot size. (a) Time evolution of the voltage, current, beam size at the sample, and power density. (b) Beam spatial profile at three different times: at the peak current, after a current drop of $10 \%$, and after a current drop of $20 \%$. The insert shows the corresponding spot sizes.

was also tested with similar results). The resolidified surfaces presented particular patterns, and the study of the structures obtained is beyond the scope of this work and will be presented in a later paper. 

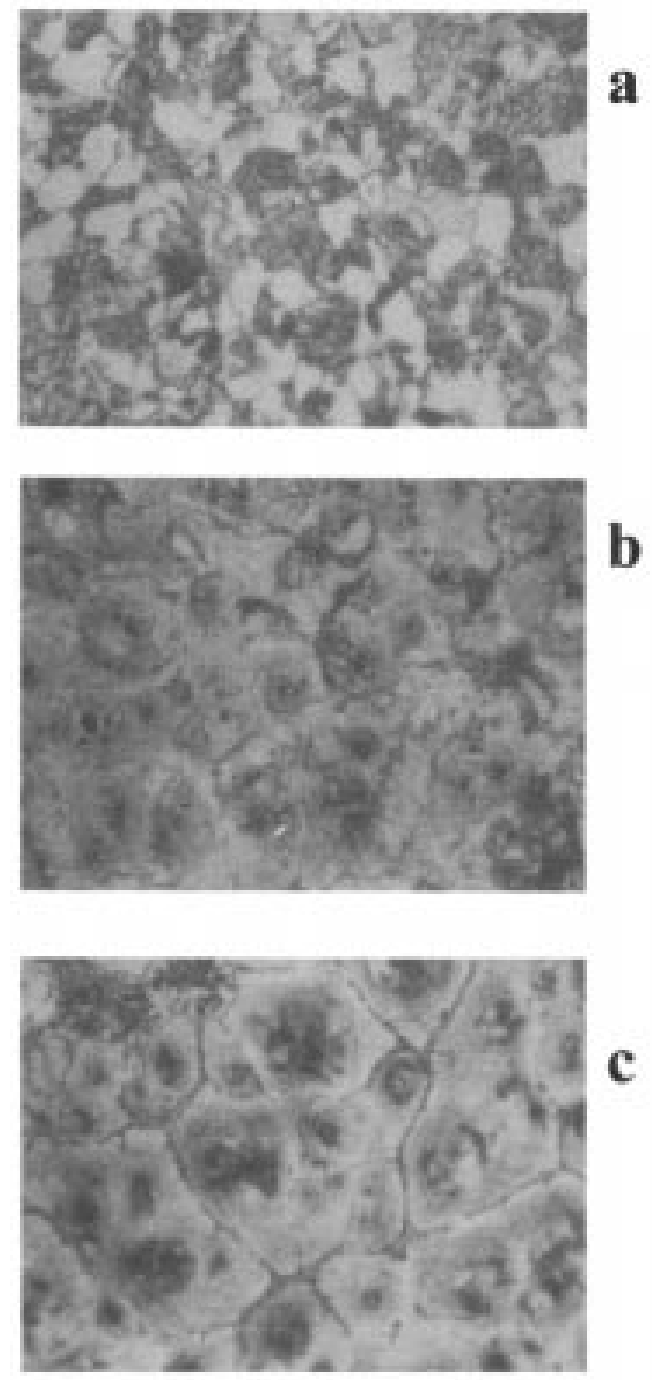

$\mathbf{5 0} \mu \mathrm{m}$

Fig. 4. Optical micrographs of the samples with identical magnification. (a) Base material before irradiation (ferrite plus pearlite). (b) Sample treated with the plane cathode with $23.5-\mathrm{kV}$ cathode voltage, 36.7-A peak current, and 35-cm cathode-sample distance (gas pressure $=260$ mtorr). (c) Sample treated with the curved cathode. Parameters $V=15 \mathrm{kV}$ and $I_{\text {peak }}=13.6 \mathrm{~A}$, distance $=16 \mathrm{~cm}$ (gas pressure $=260$ mtorr).

\section{DISCUSSION}

The numerical computations of the time evolution of the discharge voltage and current always presented a good agreement with the measured results, typically as shown in Fig. 1(b). The measured spot size of the central affected area also coincides with the predicted numerical computation, which adds confidence to the model used for the beam evolution that results in an essential tool for predicting the discharge conditions for a desired treatment and for the final interpretation of the results.

For a more precise comparison between the two samples illustrated in Fig. 4, numerical computations were performed, reproducing the same conditions used for the sample treated with the plane cathode [Fig. 4(b)]. The results are displayed in Fig. 5. Comparing that figure with Fig. 3(a), corresponding to the
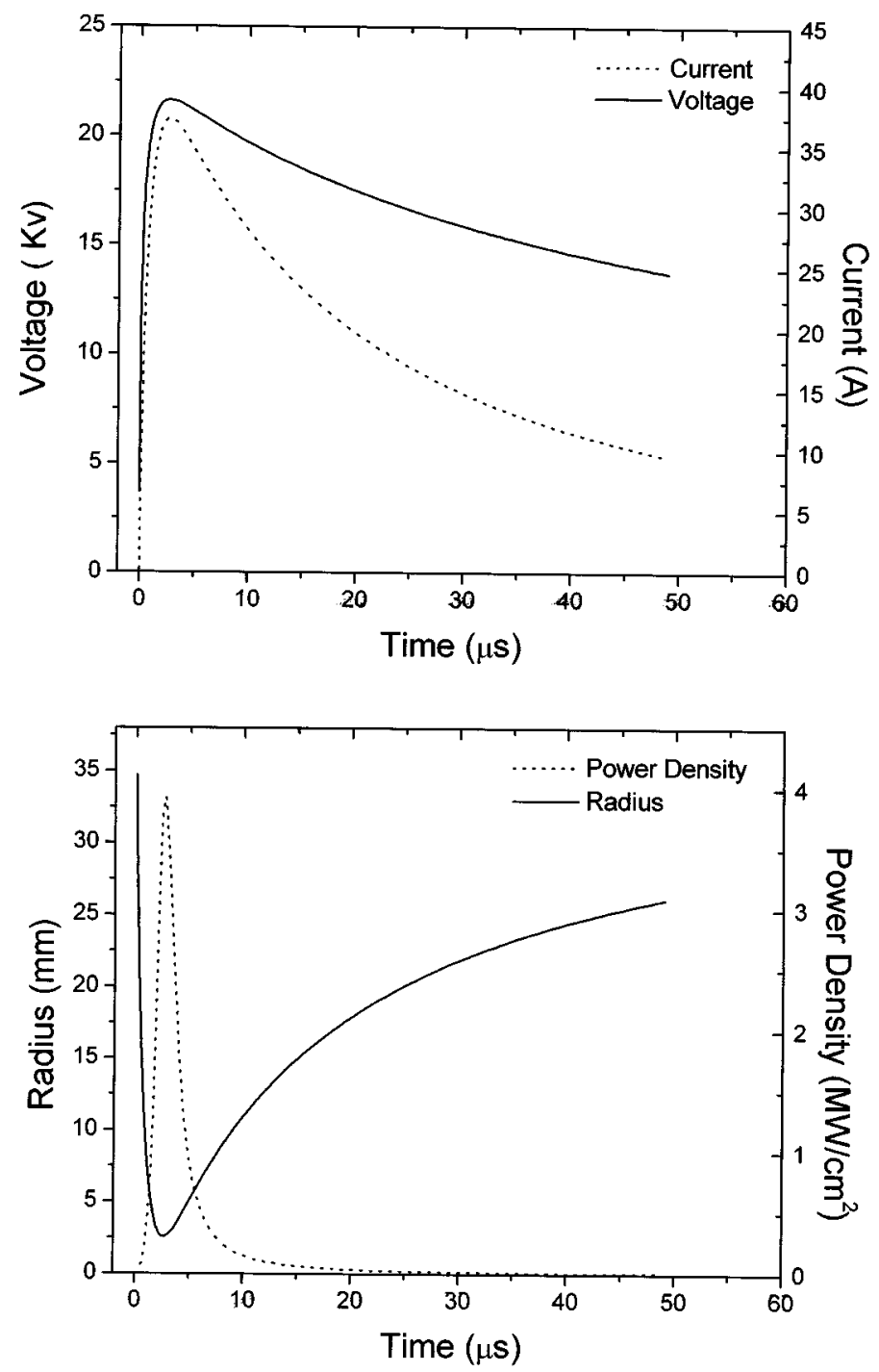

Fig. 5. Numerical simulation of the beam evolution for the plane cathode case, reproducing the conditions for the sample shown in Fig. 4(b). The sample is located $35 \mathrm{~cm}$ away from the cathode to produce the same minimum spot size. Time evolution of the voltage, current, beam size at the sample, and power density.

samples treated with the curved cathode [Fig. 4(c)], it can be noticed that the minimum spot is similar, but the plane cathode beam defocuses fast, reaching several centimeters in diameter at the power half maximum. The peak power density is more than twice that of the curved cathode beam, and the full-width half maximum of the power density is $1.7 \mu$ s for the plane cathode and $6.8 \mu \mathrm{s}$ for the curved one. The fluence at the center is lower for the plane cathode, $11 \mathrm{~J} / \mathrm{cm}^{2}$, compared with $25 \mathrm{~J} / \mathrm{cm}^{2}$ for the curved one. This result is consistent with the fact that the heat diffusion depth is proportional to the square root of the pulsewidth, and this yields a depth for the plane cathode two times smaller than the curved one. Hence, the deposited energy density is similar within a $15 \%$, consistent with the similar structures observed.

The efficiency resulted in $\varepsilon=0.085$ for the plane cathode and in $\varepsilon=0.226$ for the curved cathode. This increase in efficiency can be attributed to the increase in the ratio between the power density pulsewidth $\tau_{p}$ and the current pulsewidth $\tau_{I}$. 
In fact, for the plane cathode, $\tau_{p} / \tau_{I}=0.1$, and for the curved cathode, $\tau_{p} / \tau_{I}=0.26$, indicating that the increase in efficiency is essentially caused by the persistence of the beam without defocusing.

It should be mentioned that including the large inductance in the circuit gave rise to a rounding of the current pulse, which increased the efficiency of the plane cathode with respect to our previous experiment. In fact, without the inductance, the beam would have defocused even faster, yielding an even smaller efficiency. The numerical simulations indicate that the power density $(v \cdot i / s)$ would have had a $0.5-\mu$ s duration without the inductance, whereas with the inductance a value of $1,7 \mu \mathrm{s}$ was obtained (threefold increase in the efficiency). For the curved cathode, the same magnitude power density pulsewidth would have changed from $6.8 \mu \mathrm{s}$ with inductance to $5.4 \mu \mathrm{s}$ without it (only 25\% increase). Therefore, without the inductance, the increase in the efficiency because of the curved cathode would have been of almost 11, indicating that part of the benefits of using a concave cathode are masked by the rounding of the current pulse provided by the inductance for the plane cathode. Although the inductance had little effect in the curved cathode case, it can be predicted that a larger inductance should also provide an increase in the efficiency in this case.

\section{CONCLUSION}

The experiments and simulations show that the use of a concave cathode geometry stabilizes the focal distance of the electron beams generated by pulsed high-voltage glow discharges, providing an instrument less sensitive to sample positioning. The focal distance is in this case is essentially determined by the cathode geometry, which reduces the importance of the magnetic focusing action of the beam current. In this configuration, the evolution of the beam size is also less sensitive to the current, yielding a beam remaining focused during a longer fraction of the capacitor discharge. This result, in turn, gives rise to an increase in the electron beam deposition efficiency that is shown to be directly proportional to the increase in the power density pulsewidth. The agreement between the numerical computations and experimental results increases the confidence in the numerical predictions, providing a useful tool for to select the optimum discharge parameters and sample position for a desired treatment.

\section{ACKNOWLEDGMENT}

The contributions of H. de Rosa, F. Karlés, and F. Palumbo in the sample preparation as well as that of E. Pascualini in suggesting and providing the steel alloy used are also acknowledged.

\section{REFERENCES}

[1] W. M. Steen, Laser Material Processing, 2nd ed. London, U.K.: Springer-Verlag, 1994

[2] M. von Allmen and A. Blatter, Laser-Beam Interactions with Materials, 2nd ed. New York: Springer-Verlag, 1995.

[3] M. Riabkina-Fishman, J. Zahavi, and L. S. Zevin, "Effect of laser beam irradiation on AISI 1045 steel," J. Mater. Res., vol. 3, pp. 1108-1118, 1988.
[4] C. Hu and T. N. Baker, "Prediction of laser transformation hardening depth using a line source model," Acta Metallurg. Mater, vol. 43, no. 9, pp. 3563-3569, 1995.

[5] B. E. Wilde, M. Manohar, and C. E. Albright, "The influence of lasersurface melting on the resistance of AISI-4135 low-alloy steel to hydrogen-induced brittle-fracture," Mater. Sci. Eng. A, vol. 198, pp. 43-49, 1995.

[6] C. C. Huang, W. T. Tsai, and J. T. Lee, "Microstructure and electrochemical-behavior of laser treated $\mathrm{Fe}-\mathrm{Cr}$ and $\mathrm{Fe}-\mathrm{Cr}-\mathrm{Si}-\mathrm{N}$ surface alloyed layers on carbon-steel," Mater. Sci. Eng. A, vol. 190, pp. 199-205, 1995.

[7] R. Kralova, "Residual-stresses induced in steel by laser melting," Mater. Sci. Eng. A, vol. 174, pp. L51-L54, 1994.

[8] P. H. Steen, P. Ehrhard, and A. Schussler, "Depth of melt-pool and heataffected zone in laser-surface treatments," Metallurg. Mater. Trans. A, vol. 25A, pp. 427-435, Feb. 1994.

[9] F. Hirose, M. Takagi, H. Mori, Y. Kitoh, and T. Imura, "Microstructure of Fe-B-Si alloy surface-layers produced by laser-quenching," Jpn. J. Appl. Phys., vol. 31, pp. 3940-3945, Dec. 1992.

[10] K. Nagarathnam and K. Komvopoulos, "Microstructural characterization and in-situ transmission electron-microscopy analysis of laser-processed and thermally treated $\mathrm{Fe}-\mathrm{Cr}-\mathrm{W}-\mathrm{C}$ clad coatings," Metallurg. Trans. A, vol. 24, pp. 1621-1629, July 1993.

[11] R. A. Dugdale, "Soft vacuum processing of materials with electron beams," J. Mater. Sci., vol. 10, pp. 896-902, 1975.

[12] C. A. Moore, J. J. Rocca, G. J. Collins, P. E. Russell, and J. D. Geller, "Titanium disilicate formation by wide area electron beam irradiation," Appl. Phys. Lett., vol. 45, pp. 169-171, 1984.

[13] N. J. Ianno, J. T. Vedeyen, S. S. Chan, and B. J. Streetman, "Plasma annealing of ion implanted semiconductors," Appl. Phys. Lett., vol. 39, pp. 622-625, 1981

[14] C. A. Moore, J. J. Rocca, T. Johnson, G. J. Collins, and P. E. Russell, "Large area electron beam annealing," Appl. Phys. Lett., vol. 43, pp. 290-292, 1983.

[15] N. Mingolo and J. J. Rocca, "Production of amorphous metallic surfaces by means of a pulsed glow discharge electron beam," J. Mater. Res., vol. 7, no. 5, pp. 1096-1099, 1992

[16] H. F. Ranea-Sandoval, N. Reesor, B. T. Szapiro, C. Murray, and J. J. Rocca, "Study of intense electron beams produced by high-voltage pulsed glow discharges," IEEE Trans. Plasma Sci., vol. PS-15, pp. 361-374, Aug. 1987.

[17] J. I. Etcheverry, O. E. Martínez, and N. Mingolo, "Numerical modeling of materials processing application of a pulsed cold cathode electron gun," J. Appl. Phys., vol. 83, pp. 3856-3863, Mar. 1998.

[18] J. I. Etcheverry, N. Mingolo, J. J. Rocca, and O. E. Martínez, "A simple model of a glow discharge electron beam for materials processing," IEEE Trans. Plasma Sci., vol. 25, pp. 427-432, June 1997.

[19] N. Mingolo, C. R. González, O. E. Martínez, and J. J. Rocca, "Stabilization of a cold cathode electron beam glow discharge for surface treatment," J. Appl. Phys., vol. 82, pp. 4118-4120, Oct. 1997.

[20] J. J. Rocca, J. D. Meyer, M. R. Farrell, and G. Collins, "Glow dischargecreated electron beams: Cathode materials designs, and technological applications," J. Appl. Phys., vol. 56, pp. 790-797, Aug. 1984.

[21] K. Kobashi, S. Miyauchi, K. Miyata, K. Nishimura, and J. J. Rocca, "Etching of polycrystalline diamond films by electron beam assisted plasma," J. Mater. Res., vol. 11, pp. 2744-2748, Nov. 1996.

[22] J. D. Cobine, Gaseous Conductors. Theory and Engineering Applications. New York: Dover, 1958, p. 218.

[23] G. Francis, "The glow discharge at low pressure," in Encyclopedia of Physics, S. Flügge, Ed. Berlín, Germany: Springer-Verlag, 1956, vol. XXII, pp. 53-203.

Nélida Mingolo received the Licenciado en Física (M.S.) degree and the Ph.D. degree in physics, both from the Universidad de Buenos Aires, Argentina, in 1981, and 1992, respectively. She is Professor at the Universidad de Buenos Aires and Head of the Directed Beam Laboratory, where the electron beam treatment of metallic surfaces is being developed. She has worked mainly on amorphous metals and fast quenching techniques, as well as characterization techniques for metastable phases.

Yanina Cesa received the Licenciada en Física (M.S.) degree from the Universidad de Buenos Aires, Argentina, in 1998. She is currently pursuing the Ph.D. degree from the University de Buenos Aires in the area of phase-transition characterization techniques. 
Oscar Eduardo Martínez received the Computador Científico, the Licenciado en Física (M.S.) and the Ph.D. degrees in physics, all from Universidad de Buenos Aires, Argentina, in 1975, 1976, and 1982, respectively. He is currently a full Professor at the Universidad de Buenos Aires and a Member of the research staff of Consejo Nacional de Investigaciones Científicas y Tecnológicas de la República Argentina.

He has published more than 60 papers mainly on ultrashort pulse generation.

Dr. Martinez is a Fellow of the Optical Society of America.
Javier Ignacio Etcheverry received the Licenciado degree in mathematics and physics from the University of Buenos Aires, Argentina. He is presently pursuing the Ph.D. degree in mathematics at the University of Buenos Aires, in the area of partial differential equations.

$\mathrm{He}$ is working in mathematical modeling and numerical simulation of interactions of energetic beams with materials.

Jorge J. Rocca (S'80-M'83-SM'94) photograph and biography not available at the time of publication. 